\title{
Critical Evaluation of Nanoparticle Tracking Analysis (NTA) by NanoSight for the Measurement of Nanoparticles and Protein Aggregates
}

\author{
Vasco Filipe, ${ }^{1,2}$ Andrea Hawe, ${ }^{1}$ and Wim Jiskoot ${ }^{1,3}$
}

Received December 9, 2009; accepted January 14, 2010; published online March 4, 2010

Purpose. To evaluate the nanoparticle tracking analysis (NTA) technique, compare it with dynamic light scattering (DLS) and test its performance in characterizing drug delivery nanoparticles and protein aggregates.

Methods. Standard polystyrene beads of sizes ranging from 60 to $1,000 \mathrm{~nm}$ and physical mixtures thereof were analyzed with NTA and DLS. The influence of different ratios of particle populations was tested. Drug delivery nanoparticles and protein aggregates were analyzed by NTA and DLS. Live monitoring of heat-induced protein aggregation was performed with NTA.

Results. NTA was shown to accurately analyze the size distribution of monodisperse and polydisperse samples. Sample visualization and individual particle tracking are features that enable a thorough size distribution analysis. The presence of small amounts of large $(1,000 \mathrm{~nm})$ particles generally does not compromise the accuracy of NTA measurements, and a broad range of population ratios can easily be detected and accurately sized. NTA proved to be suitable to characterize drug delivery nanoparticles and protein aggregates, complementing DLS. Live monitoring of heat-induced protein aggregation provides information about aggregation kinetics and size of submicron aggregates.

Conclusion. NTA is a powerful characterization technique that complements DLS and is particularly valuable for analyzing polydisperse nanosized particles and protein aggregates.

KEY WORDS: dynamic light scattering; liposomes; nanoparticles; nanoparticle tracking analysis; protein aggregates.

\section{INTRODUCTION}

Most macromolecular drugs, such as proteins, peptides, DNA and RNA, cannot be administrated via the traditional oral route of administration, due to their susceptibility to enzymatic degradation or low absorption efficiency $(1,2)$. To be effective, these drugs have to be delivered in most cases via injection and/or through a drug delivery system (DDS).

DDSs not only protect the therapeutic drug or antigen from degradation and/or increase their absorption, but may also allow controlled release or the precise delivery to a specific target (3). Common examples of DDS include polymer-based particles, lipid-based carriers and virus-like particles. Given that most of these DDSs are nanosized colloidal particles, it is essential to have reliable characterization tools to ensure their quality and colloidal stability.

Another pharmaceutically important field that requires adequate tools for the analysis of particles in the nano-

\footnotetext{
$\overline{{ }^{1} \text { Division of Drug Delivery Technology, Leiden/Amsterdam Center }}$ for Drug Research, Leiden University, P.O. Box 9502, 2300 RA Leiden, The Netherlands.

${ }^{2}$ Department of Pharmaceutics, Utrecht Institute for Pharmaceutical Sciences (UIPS), Utrecht University, P.O. Box 80082, 3508 TB Utrecht, The Netherlands.

${ }^{3}$ To whom correspondence should be addressed. (e-mail: w.jiskoot@ lacdr.leidenuniv.nl)
}

meter size range is the characterization of protein aggregates. Therapeutic proteins are prone to several chemical and physical degradation pathways, which often lead to aggregation (4). The presence of aggregates in a protein formulation compromises product quality and may lead to unwanted immunogenicity (5). Thorough aggregate characterization is crucial to better understand the underlying mechanism of aggregate-related immunogenicity and ensure the quality of protein therapeutics. An accurate determination of the size and size distribution of aggregated protein formulations is not straightforward, as protein aggregates are typically very heterogeneous, with sizes ranging from a few nanometers to several micrometers (6-8). Recently, the importance of analyzing subvisible protein aggregates with sizes in the nanometer up to the low micrometer range has become recognized (9).

The most commonly used techniques for the analysis of nanoparticles and protein aggregates include dynamic light scattering (DLS), scanning electron microscopy (SEM), size exclusion chromatography (SEC), gel electrophoresis, asymmetrical flow field-flow fractionation (AF4) and analytical ultracentrifugation (AUC) (10-12). From the mentioned techniques, DLS is the most user-friendly, and it yields relatively accurate and consistent results that can be obtained in a rather short period of time (10). Therefore, DLS has become the preferred technique to routinely determine the size of nanoparticles. 
Despite being a powerful and accessible tool, DLS is also known to have several drawbacks, which are mainly inherent to the principles of the technique. Particle size is determined from fluctuations in scattered light intensity due to the Brownian movement of the particles (13). The fact that the intensity of the scattered light is proportional to the sixth power of the particle diameter makes this technique very sensitive to the presence of large particles (14). This can be an advantage if the purpose is to detect small amounts of large particles, but it can be a major drawback for accurate size determination. Dust particles or small amounts of large aggregates can impede the size determination if the main component exhibits a distinctly smaller size (15).

Nanoparticle tracking analysis (NTA), which was first commercialized in 2006, is an innovative system for sizing particles from about 30 to $1,000 \mathrm{~nm}$, with the lower detection limit being dependent on the refractive index of the nanoparticles. This technique combines laser light scattering microscopy with a charge-coupled device (CCD) camera, which enables the visualization and recording of nanoparticles in solution. The NTA software is then able to identify and track individual nanoparticles moving under Brownian motion and relates the movement to a particle size according to the following formula derived from the Stokes-Einstein Eq. I (16):

$$
\overline{(x, y)^{2}}=\frac{2 k_{B} T}{3 r_{h} \pi \eta}
$$

where $k_{B}$ is the Boltzmann constant and $\overline{(x, y)^{2}}$ is the meansquared speed of a particle at a temperature $T$, in a medium of viscosity $\eta$, with a hydrodynamic radius of $r_{h}$.

Our aim was to explore the potential of nanoparticle tracking analysis (NTA) for the analysis of nanosized particles and protein aggregates. A direct comparison with DLS was made in order to reveal the advantages and pitfalls of a technique that is now making its first steps in the field of characterization of nanoparticles and submicron protein aggregates.

\section{MATERIALS AND METHODS}

\section{Chemicals}

Poly (lactic-co-glycolic acid) 50:50 (PLGA) and 4-(2hydroxyethyl)-1-piperazine-ethanesulfonic acid (HEPES) were obtained from Sigma-Aldrich (Steinheim, Germany), chitosan (deacetylation degree 92\%, MW $120 \mathrm{kDa}$ ) from Primex (Siglufjordur, Iceland) and egg L- $\alpha$-phosphatidyl choline (EPC) from Lipoid GmbH (Ludwigshafen, Germany). 1,2-dioleoyl-snglycero-3-phospho ethanolamine (DOPE) and 1,2-dioleoyl-3trimethyl ammonium-propane (DOTAP) were supplied by INstruchemie (Delfzijl, The Netherlands). Chloroform was purchased from Biosolve (Valkenswaard, The Netherlands). All other chemicals used were from Sigma-Aldrich (Steinheim, Germany), unless mentioned otherwise.

\section{Preparation of Polystyrene Bead Samples}

Polystyrene nanometer standard beads with sizes of 60 , 100, 200, 400 and 1,000 $\mathrm{nm}$ were purchased from Thermo Scientific (Fremont, USA). They were diluted from the supplied package in deionized water until the concentration was acceptable for NTA measurements, i.e. between $10^{7}$ and $10^{9}$ total particles $/ \mathrm{ml}$. Thus, from the supplier's recipient, a 1:30,000 volume based dilution was made for the 60 -nm beads, 1:100,000 dilution for the 100-nm beads, 1:25,000 dilution for the 200 -nm beads, 1:2,500 dilution for the $400-\mathrm{nm}$ beads, and 1:100 dilution for the 1,000-nm beads. All polystyrene bead measurements were performed with these samples, either alone or mixed at different volume ratios or number ratios based on NTA particle counts, as stated in the results section.

The $100-\mathrm{nm}$ and $400-\mathrm{nm}$ beads mixture used for the spiking experiments contained about $1.7 * 10^{8}$ beads $/ \mathrm{ml}$. For these experiments, 2 or $40 \mu \mathrm{l}$ of a suspension of $1,000-\mathrm{nm}$ beads (ca. $1.6^{*} 10^{8}$ particles $/ \mathrm{ml}$ ) were added to $500 \mu \mathrm{l}$ of the $100-\mathrm{nm}$ and $400-\mathrm{nm}$ beads mixture, which resulted in a $1,000-\mathrm{nm}$ beads concentration of about $6.4 * 10^{5}$ beads/ $/ \mathrm{ml}$ and $1.2 * 10^{7}$ beads $/ \mathrm{ml}$, respectively. The resulting number ratios of 1,000-nm beads to the beads in the initial mixture was 1:267 for the $2 \mu \mathrm{l}$ spike (small spike) and 1:13 for the $40 \mu \mathrm{l}$ spike (big spike).

\section{Preparation of Drug Delivery Nanoparticles}

N-trimethyl chitosan (TMC) with a degree of quaternization of $15 \%$ was prepared from chitosan and used to make TMC nanoparticles, as described in the literature (17). In short, TMC was dissolved in a $5 \mathrm{mM}$ HEPES buffer ( $\mathrm{pH}$ 7.4), and pentasodium tripolyphosphate (TPP) was added under continuous stirring to a weight ratio TMC:TPP of 10:1.8. Nanoparticles were collected by centrifugation $(30 \mathrm{~min}$, $15,000 \mathrm{~g}$ ) on a glycerol bed, to avoid aggregation, and resuspended in $5 \mathrm{mM}$ HEPES buffer ( $\mathrm{pH}$ 7.4). The sample was diluted 1,000-fold with deionized water before the measurements.

PLGA nanoparticles were prepared by an "oil-in-water" solvent evaporation method, using polysorbate 20 as emulsifying agent. Briefly, $1 \mathrm{ml}$ of dichloromethane containing $50 \mathrm{mg}$ of PLGA and $2 \mathrm{ml} \mathrm{1 \% (w/v)} \mathrm{polysorbate} 20$ were emulsified using an ultrasonic processor for $15 \mathrm{~s}$ at $70 \mathrm{~W}$ (Branson Instruments, Connecticut, USA). The emulsion was transferred to $50 \mathrm{ml}$ of $0.02 \%(\mathrm{w} / \mathrm{v})$ polysorbate 20 in water and stirred at $50^{\circ} \mathrm{C}$ for $1 \mathrm{hr}$. The resulting PLGA nanoparticles were collected by centrifugation $(8,000 \mathrm{~g}$ for $10 \mathrm{~min})$ and washed twice in distilled water to remove excess polysorbate 20 . The sample was diluted 2000 -fold with deionized water before the measurements.

Cationic liposomes were prepared by the film-hydrationrehydration method and sized by sonication. In detail, a lipid film was formed by solvent evaporation of a chloroform solution of EPC, DOPE and DOTAP in a rotary evaporator at $37^{\circ} \mathrm{C}$. To prepare $1 \mathrm{ml}$ of liposome dispersion, a total amount of $28 \mu \mathrm{mol}$ lipid was used at a EPC/ DOPE/DOTAP molar ratio of $4 / 2 / 1$. The film was hydrated

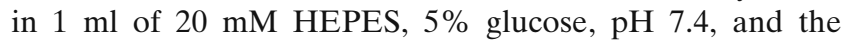
dispersion was equilibrated for $1 \mathrm{hr}$ at room temperature. The dispersion was then sonicated twice for $30 \mathrm{~s}$, with $30 \mathrm{~s}$ interval, using a Branson Sonifier 250 (Branson Ultrasonics, Danbury, UK), with $3 \mathrm{~mm}$ microtip at $20 \mathrm{~mW}$ energy output. The sample was diluted 10,000-fold with deionized water before the measurements. 
All the buffers used in this section were filtered using a $0.22-\mu \mathrm{m}$ PES low binding syringe-driven filter unit (Millex ${ }^{\mathrm{TM}}$ GP, Millipore, Ireland), and the absence/very low content of submicron particles was confirmed by their visualization in the NanoSight sample chamber.

\section{Preparation of Protein Aggregates}

A recombinant human monoclonal antibody of the $\mathrm{IgG}_{1}$ subclass ( $\mathrm{IgG})$ was used for preparing $\mathrm{IgG}$ aggregates. The $\mathrm{IgG}$ was formulated at a concentration of $1.0 \mathrm{mg} / \mathrm{ml}$ in $10 \mathrm{mM}$ sodium citrate (Merck, Darmstadt, Germany), 5\% (w/v) sucrose (Sigma-Aldrich, Buchs, Switzerland), $\mathrm{pH}$ 6.0. The $\mathrm{IgG}$ formulation was filtered using a $0.22-\mu \mathrm{m}$ PES low binding syringe-driven filter unit. One $\mathrm{ml}$ of the filtered $\mathrm{IgG}$ formulation was placed in 1.5-ml reaction tubes (Eppendorf, Hamburg, Germany) and incubated for $15 \mathrm{~min}$ at $74^{\circ} \mathrm{C}$ in a heating block (Eppendorf, Hamburg, Germany). The sample was diluted 50 -fold with the formulation buffer before each measurement.

Recombinant human insulin (insulin) was formulated in $10 \mathrm{mM}$ sodium phosphate, $\mathrm{pH} 7.4$, and the formulation was filtered using the same filter unit as for the $\mathrm{IgG}$ formulation. Insulin aggregation was induced via a metal-catalyzed oxidation reaction by the addition of copper chloride $(0.04 \mathrm{mM})$, followed by ascorbic acid $(4 \mathrm{mM})$. The formulation was incubated at room temperature for three hours, and the reaction was stopped by the addition of $1 \mathrm{mM}$ ethylenediaminetetraacetic acid (EDTA).

All the buffers used in this section were filtered using a $0.22-\mu \mathrm{m}$ PES low binding syringe-driven filter unit, and the absence/very low content of submicron particles was confirmed by their visualization in the NanoSight sample chamber.

\section{Dynamic Light Scattering (DLS)}

DLS measurements were performed with a Malvern Zetasizer Nano ZS (Malvern, Herrenberg, Germany) equipped with a 633-nm He-Ne laser and operating at an angle of $173^{\circ}$. The software used to collect and analyze the data was the Dispersion Technology Software version 6.01 from Malvern. Five-hundred $\mu \mathrm{l}$ of each sample was measured in single-use polystyrene half-micro cuvettes (Fisher Emergo, Landsmeer, The Netherlands) with a pathlength of $10 \mathrm{~mm}$.

The measurements were made at a position of $4.65 \mathrm{~mm}$ from the cuvette wall with an automatic attenuator and at a controlled temperature of $25^{\circ} \mathrm{C}$. For each sample, 15 runs of $10 \mathrm{~s}$ were performed, with three repetitions for all the polystyrene beads and six repetitions for the polymer nanoparticles, liposomes and protein aggregates. The intensity size distribution, the Z-average diameter (Z-ave) and the polydispersity index $(\mathrm{PdI})$ were obtained from the autocorrelation function using the "general purpose mode" for the monodisperse polystyrene beads, liposomes and polymer samples, the "multiple narrow mode" for the mixtures of polystyrene beads and the "protein analysis mode" for the protein aggregates. The default filter factor of $50 \%$ and the default lower threshold of 0.05 and upper threshold of 0.01 were used. The error bars displayed on the DLS graphs were obtained by the standard deviation (SD) of three or six measurements of the same sample.

\section{Nanoparticle Tracking Analysis (NTA)}

NTA measurements were performed with a NanoSight LM20 (NanoSight, Amesbury, United Kingdom), equipped with a sample chamber with a 640-nm laser and a Viton fluoroelastomer O-ring. The samples were injected in the sample chamber with sterile syringes (BD Discardit II, New Jersey, USA) until the liquid reached the tip of the nozzle. All measurements were performed at room temperature except the live monitoring protein heat stress measurements (see section below).

The software used for capturing and analyzing the data was the NTA 2.0 Build 127. The samples were measured for $40 \mathrm{~s}$ with manual shutter and gain adjustments. The "single shutter and gain mode" was used to capture the monodisperse polystyrene beads, the $60 / 100 \mathrm{~nm}$ beads mixture, the liposomes, the TMC particles and the protein aggregates. The "extended dynamic range mode," which splits the capture video into two videos with independent shutter and gain settings, was used for all the other mixtures of monodisperse polystyrene beads, the PLGA particles and the insulin aggregates. Three measurements of the same sample were performed for all the polystyrene beads and six measurements for the polymer nanoparticles and protein aggregates. The error bars displayed on the NTA graphs were obtained by the standard deviation of the different measurements of each sample. The mean size and SD values obtained by the NTA software correspond to the arithmetic values calculated with the sizes of all the particles analyzed by the software.

\section{Live Monitoring of Protein Heat Stress}

For the live monitoring of protein aggregation, the above-mentioned $\operatorname{IgG}$ was formulated at a concentration of $1.0 \mathrm{mg} / \mathrm{ml}$ in $100 \mathrm{mM}$ sodium citrate (Merck, Darmstadt, Germany), $\mathrm{pH}$ 7.6. Unstressed $\mathrm{IgG}$ was inserted in the NanoSight sample chamber at room temperature, and the heating block was then set to $50^{\circ} \mathrm{C}$. Once the chamber had reached the set temperature, which took about $10 \mathrm{~min}$, a video was recorded for $40 \mathrm{~s}\left(\mathrm{t}_{0}\right)$, followed by three time points with the same video length, at 15, 25 and $35 \mathrm{~min}$. The videos of the first three time points were captured with the "single shutter and gain mode" and of the last time point with the "extended dynamic range mode," because of the high sample polydispersity observed for this time point.

\section{RESULTS AND DISCUSSION}

\section{Evaluation of NTA Performance and Comparison to DLS}

\section{Monodisperse Polystyrene Beads}

In order to verify the accuracy of NTA to size monodisperse samples, standard polystyrene beads of $60 \mathrm{~nm}, 100 \mathrm{~nm}, 200 \mathrm{~nm}, 400 \mathrm{~nm}$ and 1,000 nm were analyzed with NTA, and the results were compared to DLS (Fig. 1). While NTA requires particle concentrations of $10^{7}-10^{9} / \mathrm{ml}$, the DLS concentration range is less critical and depends upon a number of instrumental and sample properties (18). For most of the samples used in this study, the DLS concentration was about $10^{8}-10^{12}$ particles $/ \mathrm{ml}$ (data not shown). Given the 
$60 \mathrm{~nm}$ beads

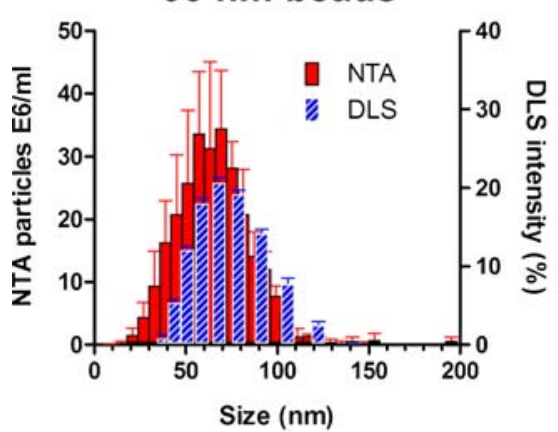

$100 \mathrm{~nm}$ beads

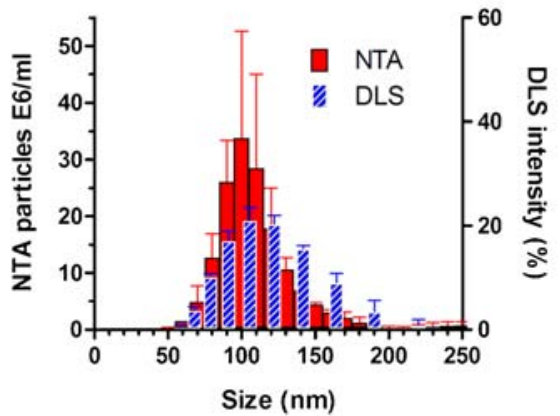

$200 \mathrm{~nm}$ beads

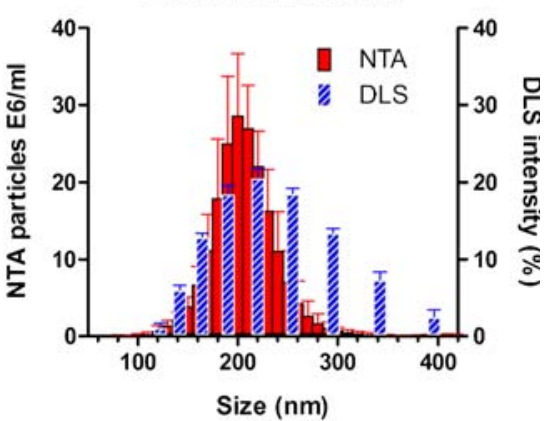

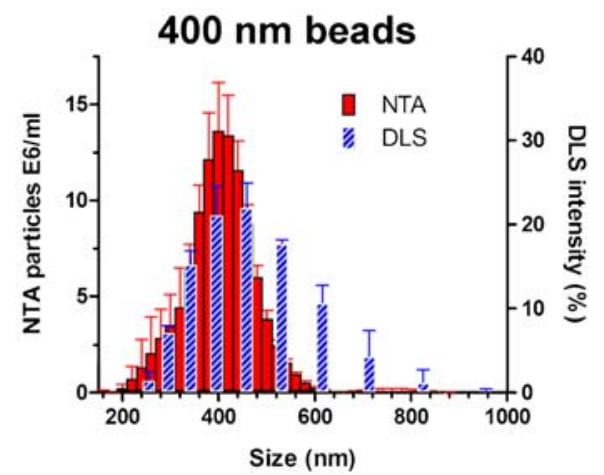

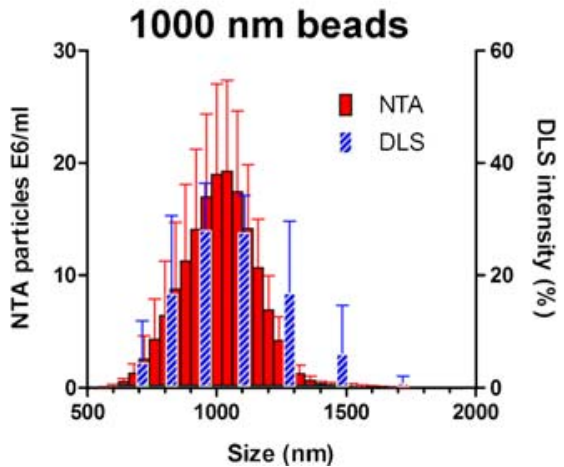

Fig. 1. Size distribution from NTA and DLS measurements of monodisperse polystyrene beads. Error bars represent standard deviations obtained from three measurements of the same sample.

difference in concentration range between the two techniques, a concentration suitable for both techniques was selected for each bead size.

Contrary to DLS, NTA enables sample visualization and provides approximate particle concentrations, which are very useful features. Both techniques showed good sizing accuracy and relatively narrow distributions for all monodisperse samples. Nevertheless, it is possible to observe a tailing of all DLS size distributions towards larger sizes, mostly due to the immense contribution of a few large particles to the overall scattering (14).

The mean size values obtained by NTA are slightly smaller and closer to the expected values than the $\mathrm{Z}$-ave given by DLS, but all values are close to the bead size specified by the manufacturer (Table I). However, the error bars of the size distribution obtained for each sample are smaller with DLS (Fig. 1), which is a consequence of the large amount of statistical data collected by DLS when compared to NTA. In fact, these high error bars in the NTA results are mostly caused by different particle counts between each measurement. The size distributions are practically the same, but the software sometimes detects slightly more or slightly less particles between each measurement of the same sample. This variation in the number of particles detected by NTA brings attention to the imprecision of the particle concentration given by this technique. Still, though not the primary aim of NTA, its capability to provide approximate submicron particle counts is an obvious advantage of the method over DLS.

While DLS measurements are fast and rather straightforward, NTA requires several optimization steps by a skilled operator, e.g. with respect to indentifying suitable settings for the video capture and analysis. Whereas DLS can automatically adjust the attenuator to adapt to a wide range of sample concentrations, the search for the right sample concentration for a successful NTA measurement can be time-consuming, as it may require various dilution steps. However, NTA proved to be slightly more accurate than DLS for sizing monodisperse samples.

Table I. Mean Size and Size Distribution of Monodisperse Polystyrene Beads from NTA and DLS Measurements

\begin{tabular}{|c|c|c|c|c|c|}
\hline \multirow[b]{2}{*}{ Bead size $(\mathrm{nm})$} & \multicolumn{2}{|c|}{ DLS } & \multicolumn{3}{|c|}{ NTA } \\
\hline & Z-ave (nm) & PdI & Mean $(\mathrm{nm})$ & $\mathrm{SD}(\mathrm{nm})$ & Particle conc. $(\mathrm{E} 8 / \mathrm{ml})$ \\
\hline 60 & $68 \pm 1$ & $0.04 \pm 0.01$ & $66 \pm 2$ & $20 \pm 1$ & 7.70 \\
\hline 100 & $112 \pm 4$ & $0.13 \pm 0.07$ & $105 \pm 6$ & $30 \pm 10$ & 1.87 \\
\hline 200 & $218 \pm 1$ & $0.04 \pm 0.01$ & $200 \pm 5$ & $30 \pm 5$ & 2.32 \\
\hline 400 & $443 \pm 5$ & $0.13 \pm 0.03$ & $394 \pm 7$ & $62 \pm 6$ & 1.08 \\
\hline 1,000 & $1056 \pm 164$ & $0.36 \pm 0.08$ & $989 \pm 51$ & $117 \pm 14$ & 1.64 \\
\hline
\end{tabular}

$Z$-ave Z-average; $P d I$ polydispersity index; $S D$ standard deviation calculated by the NTA software; Conc. concentration in particles E8/ml as measured by NTA. Numbers represent average values \pm standard deviation ( $n=3$ measurements). See Materials and Methods for details. 
Mixtures of Monodisperse Polystyrene Beads: Fixed Number Ratio

One of the well-known pitfalls of DLS is its low peak resolution, i.e. it can only resolve particle populations that differ in size at least by a factor of 3 (19). Thus, with the purpose of testing the resolution of NTA, the monodisperse polystyrene standard beads analyzed in the previous section were mixed at a fixed number ratio $(60 \mathrm{~nm}$ and $100 \mathrm{~nm} ; 100 \mathrm{~nm}$ and $200 \mathrm{~nm} ; 200 \mathrm{~nm}$ and $400 \mathrm{~nm} ; 400 \mathrm{~nm}$ and 1,000 nm) and analyzed with both techniques. The two-dimensional (2D) size

\section{a) 60 and $100 \mathrm{~nm}$ beads}

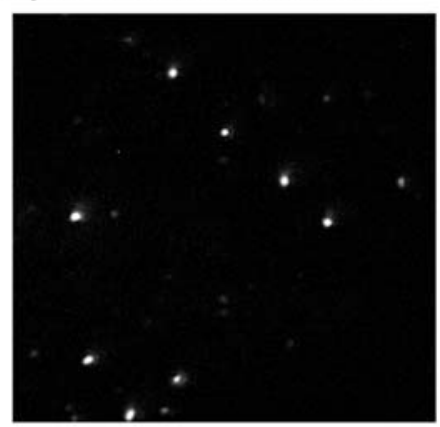

\section{b) 100 and $200 \mathrm{~nm}$ beads}

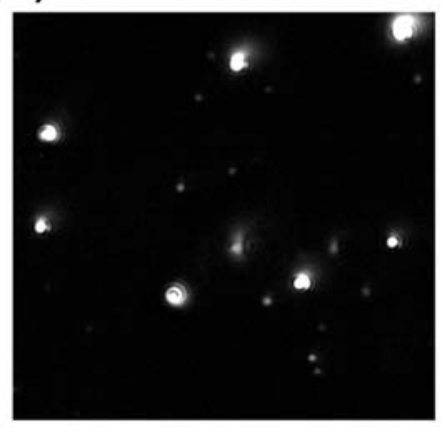

C) 200 and $400 \mathrm{~nm}$ beads

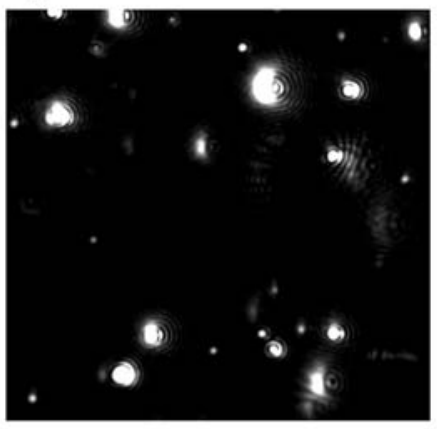

\section{d) 400 and $1000 \mathrm{~nm}$ beads}

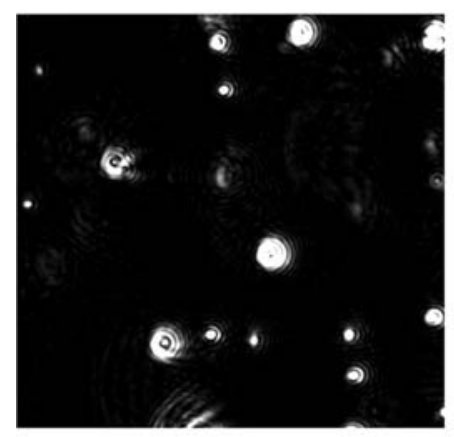

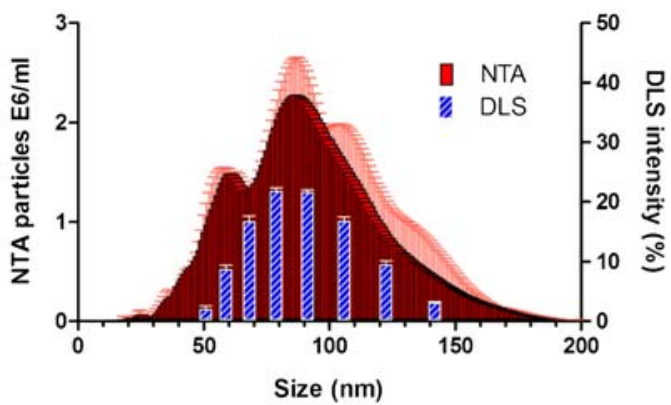
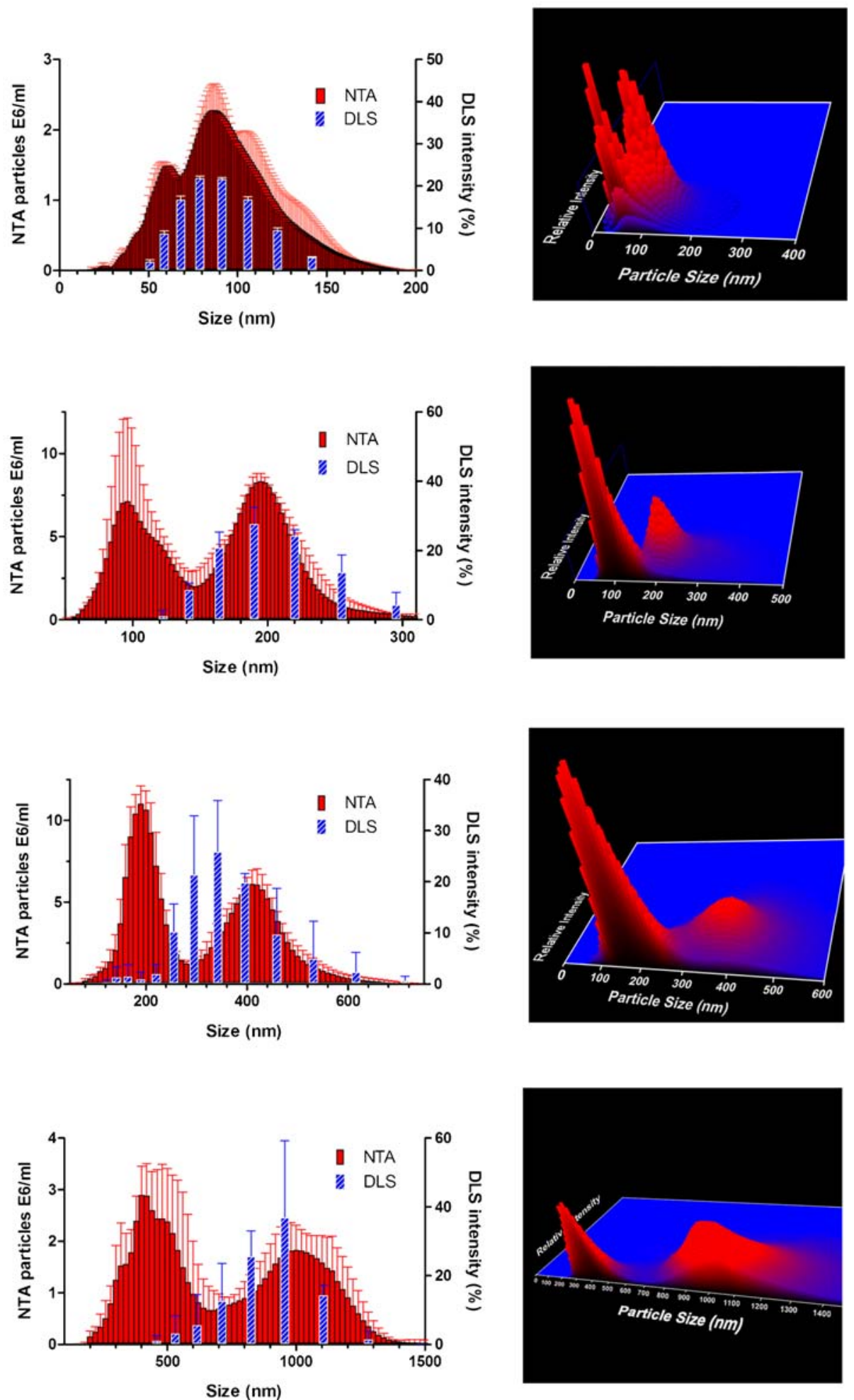

Fig. 2. Size distribution from NTA and DLS measurements of mixtures of monodisperse polystyrene beads (middle panels) with the corresponding NTA video frame (left panels) and 3D graph (size $v s$. intensity $v$ s. concentration; right panels). a) 60-nm/100-nm beads at a 4:1 number ratio; b) 100$\mathrm{nm} / 200-\mathrm{nm}$ beads at a 1:1 number ratio; c) 200-nm/400-nm beads at a 2:1 number ratio; d) 400-nm/1,000-nm beads at a 1:1 number ratio. 
Table II. Mean Size and Size Distribution of Mixtures of Monodisperse Polystyrene Beads from NTA and DLS Measurements

\begin{tabular}{|c|c|c|c|c|c|c|c|c|}
\hline \multirow[b]{2}{*}{ Bead size (nm) } & \multicolumn{3}{|c|}{ DLS } & \multicolumn{5}{|c|}{ NTA } \\
\hline & Z-ave (nm) & $\mathrm{PdI}$ & Peak (nm) & Mean (nm) & $\mathrm{SD}(\mathrm{nm})$ & Peak 1 (nm) & Peak $2(\mathrm{~nm})$ & Peak $3(\mathrm{~nm})$ \\
\hline $60+100$ & $84 \pm 1$ & $0.08 \pm 0.01$ & $83 \pm 7$ & $90 \pm 3$ & $33 \pm 4$ & $58 \pm 4$ & $91 \pm 9$ & - \\
\hline $100+200$ & $195 \pm 2$ & $0.40 \pm 0.20$ & $194 \pm 5$ & $162 \pm 13$ & $60 \pm 6$ & $98 \pm 2$ & $196 \pm 2$ & - \\
\hline $200+400$ & $347 \pm 42$ & $0.19 \pm 0.03$ & $359 \pm 32$ & $298 \pm 20$ & $122 \pm 9$ & $195 \pm 5$ & $410 \pm 10$ & - \\
\hline $400+1,000$ & $952 \pm 31$ & $0.26 \pm 0.05$ & $912 \pm 75$ & $712 \pm 36$ & $296 \pm 20$ & $427 \pm 46$ & $1067 \pm 94$ & - \\
\hline $100+400$ & $430 \pm 34$ & $0.29 \pm 0.08$ & $363 \pm 29$ & $265 \pm 16$ & $157 \pm 11$ & $98 \pm 5$ & $419 \pm 12$ & - \\
\hline $100+400+$ S. Spike & $467 \pm 22$ & $0.35 \pm 0.05$ & $378 \pm 32$ & $338 \pm 36$ & $163 \pm 23$ & $99 \pm 10$ & $384 \pm 12$ & $850 \pm 83$ \\
\hline $100+400+$ B. Spike & $698 \pm 26$ & $0.14 \pm 0.07$ & $750 \pm 65$ & $527 \pm 101$ & $353 \pm 11$ & $106 \pm 4$ & $420 \pm 22$ & $997 \pm 31$ \\
\hline
\end{tabular}

Z-ave Z-average; $P d I$ polydispersity index; $S D$ standard deviation calculated by the NTA software; $S$. Spike small spike with 1,000-nm beads to a $100-\mathrm{nm} / 400-\mathrm{nm}$ beads mixture; $B$. Spike big spike with $1,000 \mathrm{~nm}$ beads to a $100 \mathrm{~nm}$ and $400 \mathrm{~nm}$ beads mixture. Numbers represent average values \pm standard deviation ( $n=3$ measurements). See Materials and Methods for details. The peaks correspond to the highest value observed for a certain size.

distributions of DLS and NTA, with the corresponding NTA video frames and three-dimensional (3D) graphs (size vs. intensity $v s$. concentration) are shown in Fig. 2.

From these results, the difficulty of DLS in resolving peaks of polydisperse samples becomes apparent, as it was not possible to separate the two bead sizes of any of the mixtures. On the other hand, NTA was able to resolve and distinguish the two size populations in all mixtures and yielded accurate size estimations of the beads in the mixtures (Table II). The 2D size distributions show that DLS only gives a single peak for the mixtures shifted towards the larger particle size present, which is again related to its bias to larger particles. The error bars of the DLS results of the two mixtures with the larger bead size (Fig. 2c and d) are larger than the ones of the NTA results. This is related to the difficulty that the DLS software has to fit the data of an autocorrelation curve of a sample that has two populations with size differences smaller than the peak resolution limit of this technique. As a result, the single peak as calculated by the DLS software is prone to changes in shape and position from measurement to measurement, giving rise to relatively large error bars in the average result.

The two different bead sizes with different scattering intensities can be observed in the NTA video frames and 2D size distribution graphs and can be clearly distinguished in the 3D graphs (Fig. 2). Despite having a $60-\mathrm{nm} / 100-\mathrm{nm}$ bead number ratio of 4:1 (Fig 2a), NTA analysis of this mixture shows more 100 -nm beads than 60 -nm beads. This is mainly caused by a masking effect of the larger beads over the smaller beads, explained in detail in the Influence of Small Numbers of Large Particles section, combined with the fact that some of the $60-\mathrm{nm}$ beads move so fast that they often move out of focus in the detection area before they can be tracked long enough to be considered for the final result. Nevertheless, the difference in scattering intensities displayed by the $3 \mathrm{D}$ graphs proves to be very useful to confirm the presence of different populations of similar sizes, such as in this $60-\mathrm{nm} / 100-\mathrm{nm}$ bead mixture. While the $2 \mathrm{D}$ graph shows a peak at $100 \mathrm{~nm}$ and a shoulder at $60 \mathrm{~nm}$, the 3D graph shows two distinct size populations, clearly confirmed by the higher light scattering intensity of the $100-\mathrm{nm}$ particles compared to the $60-\mathrm{nm}$ ones. Thereby, the third dimension (scattering intensity) in NTA contributes not only to the resolution of particle populations, but also provides information about the nature of the particles: for particles with equal refractive index, the larger ones should scatter more light, proportional to the diameter to the power six.

Measuring the mixture of monodisperse beads with DLS is as easy and fast as measuring the standard polystyrene beads alone, but the results do not reflect the samples' real content. On the other hand, NTA analysis of two different particle sizes implies in most cases the use of the "extended dynamic range mode," which adds a complex variable to the analysis. This mode allows the recording of a set of two videos at the same time with different shutter and gain settings, enabling the simultaneous analysis of large and small particles in one measurement. A big advantage of NTA is the unbiased high peak resolution for polydisperse samples, which is not possible by DLS.

\section{Mixtures of Monodisperse Polystyrene Beads: Effect of Number Ratio}

In the field of nanoparticle characterization, it is important to have tools that are able to detect and characterize small amounts of a certain particle size population, different from the main population. Thus, to elucidate the ratio detection limits of NTA, 100 and $400 \mathrm{~nm}$ polystyrene beads were mixed at 100-nm/400-nm bead number ratios of 3:1, 6:1, 15:1, 150:1 and 300:1, and analyzed with NTA and DLS (Fig. 3). The ratios were based on the particle concentration of the individual bead samples obtained by NTA.

The selection of the distinct sizes of beads for these measurements took into consideration the DLS theoretical peak resolution of 3:1 in size. Nonetheless, DLS was not able to distinguish the two bead sizes for the lower 100-nm/400-nm bead number ratios of 3:1 and 6:1, for which mainly the larger beads were detected. When the number ratio reached 300:1, the larger particles were no longer shown by DLS.

On the other hand, analysis with NTA enabled accurate sizing and a clear distinction of the two size populations for all the ratios analyzed. The presence of the two distinct size populations is very clear in the video frames of Fig. 3. In fact, being able to see the sample and search for the desired location where the video is recorded enables the operator the choice of including or excluding certain particles. Therefore, the appearance of the $400 \mathrm{~nm}$ peak for ratios bigger than 300:1 depends on the operator. Being able to see and scan the sample is a useful feature of NTA, but it should be used prudently to avoid false or biased results. 


\section{$3: 1$ ratio}

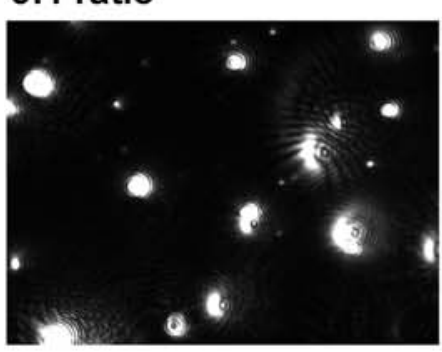

\section{$6: 1$ ratio}
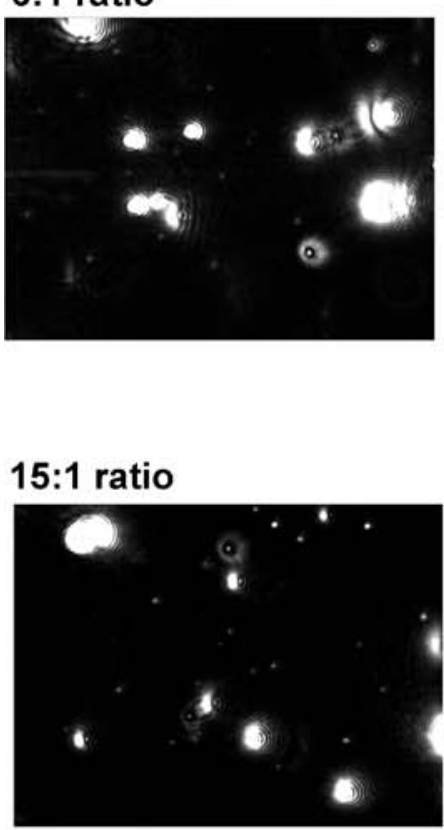

150:1 ratio
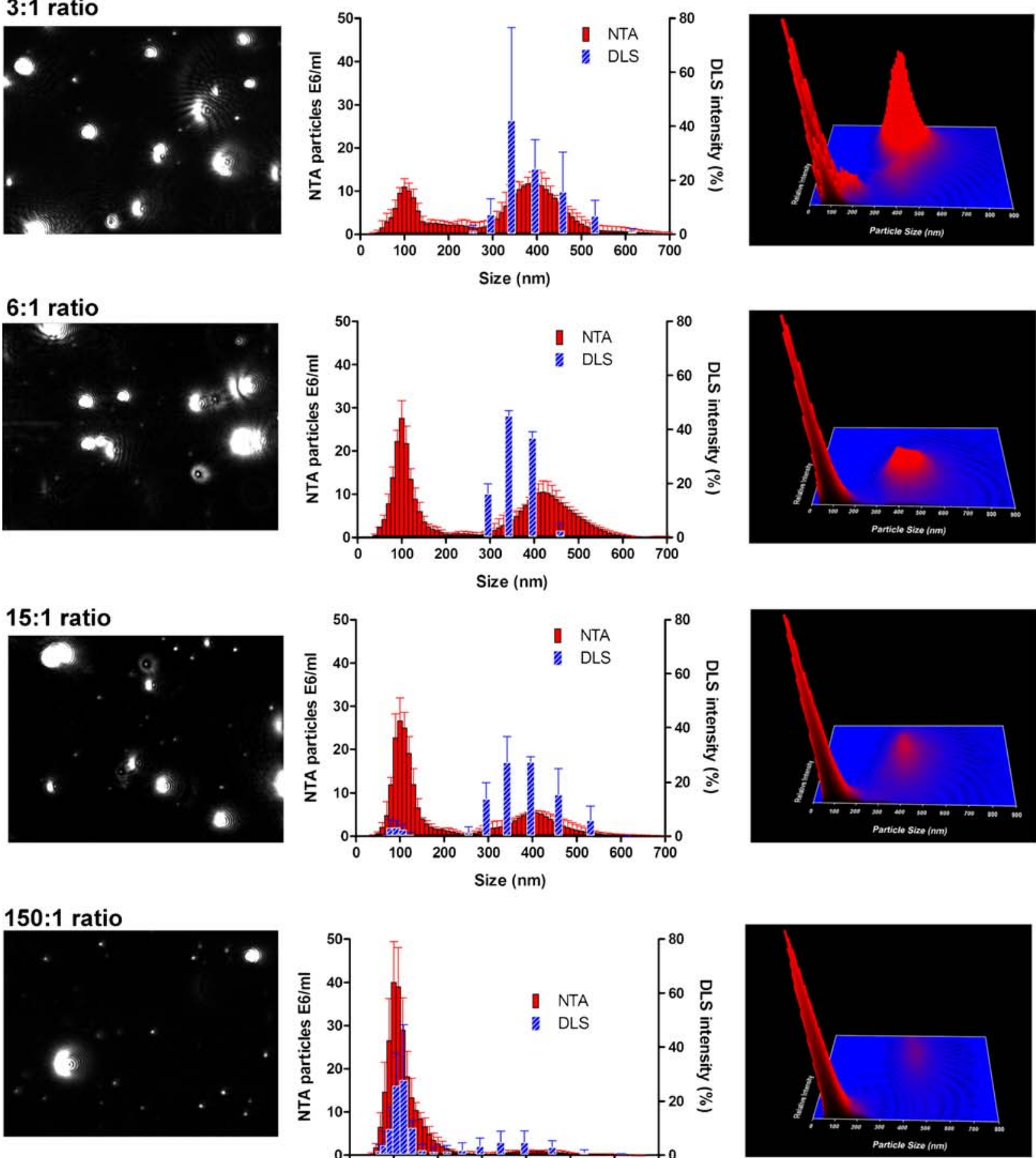

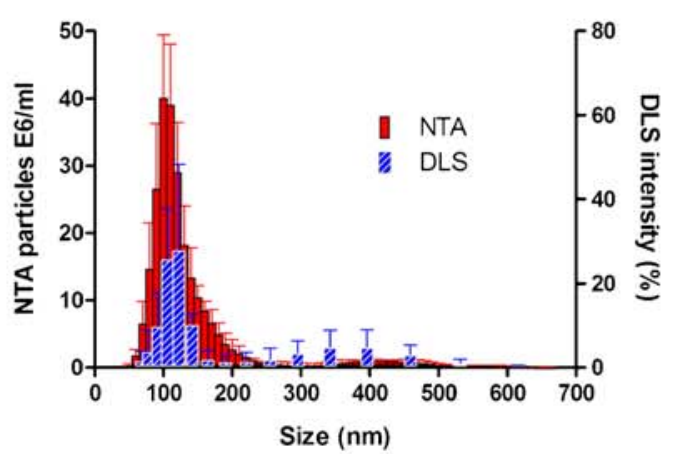

$300: 1$ ratio
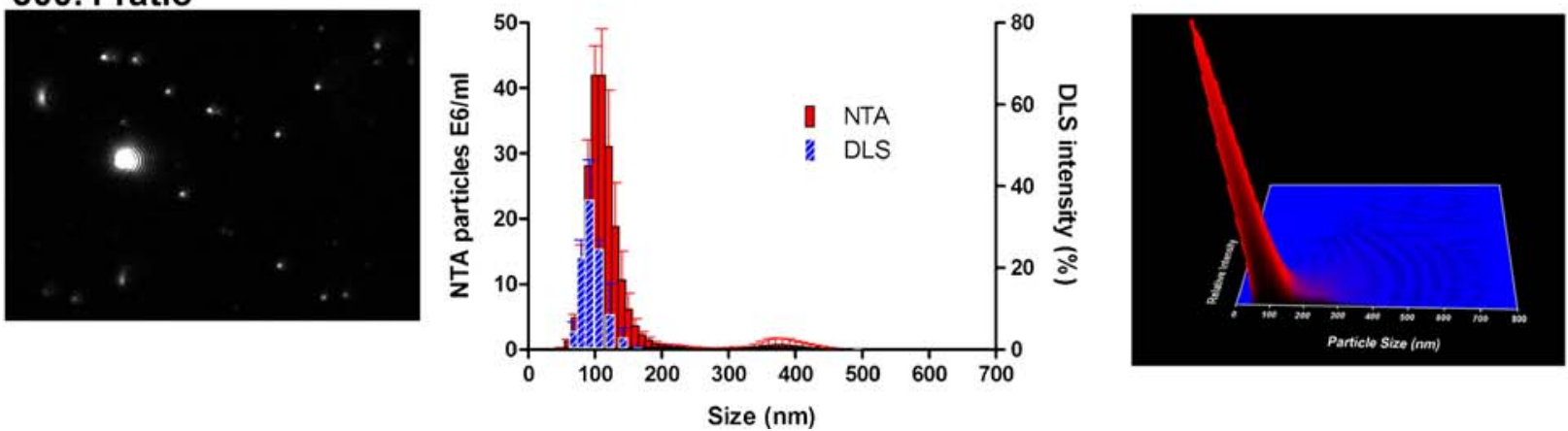
Fig. 3. Influence of different number ratios of $100-\mathrm{nm} / 400-\mathrm{nm}$ monodisperse beads in NTA and DLS measurements (middle panels) with the corresponding NTA video frame (left panels) and normalized 3D graph (size vs. intensity vs. concentration; right panels).

\section{Influence of Small Numbers of Large Particles}

One of the main concerns of DLS is the influence that a small amount of large particles, such as dust, may have on the outcome. The NTA technique is based on the tracking of single particles, whereas DLS measures a bulk of particles with a strong bias to the largest particles present in the sample. Therefore, the performance of NTA is expected to be less sensitive than DLS to the presence of minute amounts of large particles. To compare the influence of large particles on NTA and DLS results, a mixture of $100-\mathrm{nm}$ and $400-\mathrm{nm}$ polystyrene beads was spiked with two different amounts of 1,000-nm beads. The resulting number ratios of 1,000-nm beads to the beads in the initial mixture was 1:267 for the small spike and 1:13 for the big spike (Fig. 4).

The small spike was sufficient to cause an increase of about $40 \mathrm{~nm}$ in the $\mathrm{Z}$-ave and of about 0.05 in the PdI determined by DLS (Table II). The same spike made the NTA analysis slightly more complicated because the highly scattering 1,000-nm beads made the $100-\mathrm{nm}$ beads slightly more difficult to detect. However, with optimized settings (Table III), all bead types present in the sample could be detected and accurately sized by NTA (Fig. 4b). Nevertheless, after the spike, the number of 100-nm beads detected by NTA decreased by about $70 \%$ and the number of $400-\mathrm{nm}$ beads by about $20 \%$. The intense light scattering of large particles makes the small particles more difficult to detect and prevents some of them from being tracked by the NTA software.

The big spike increased the Z-ave of DLS from 430 to $698 \mathrm{~nm}$, but curiously decreased the PdI from 0.29 to 0.14 .

\section{a) Unspiked}
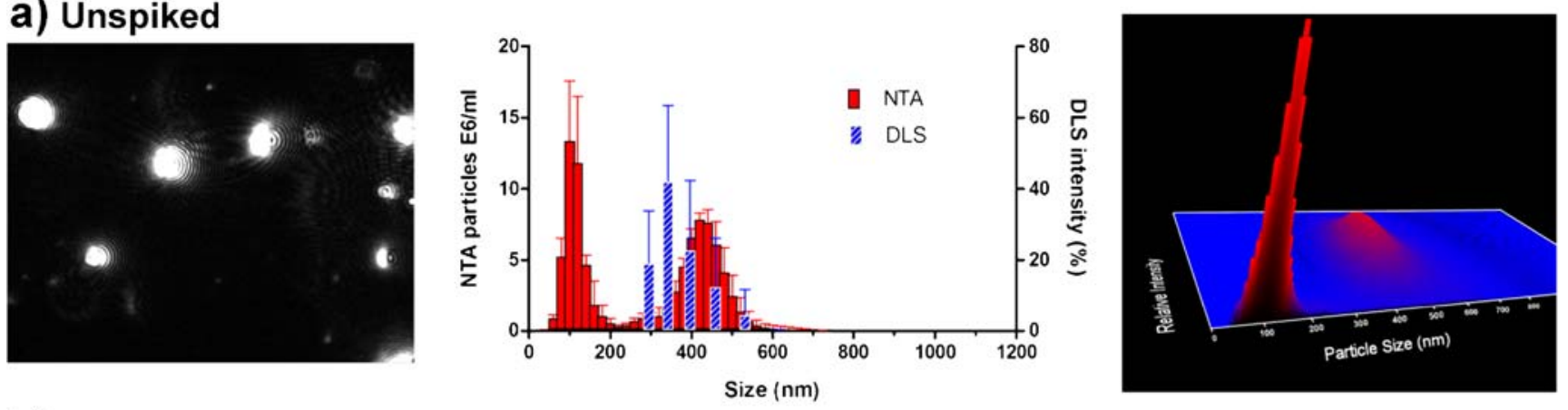

\section{b) Small spike}
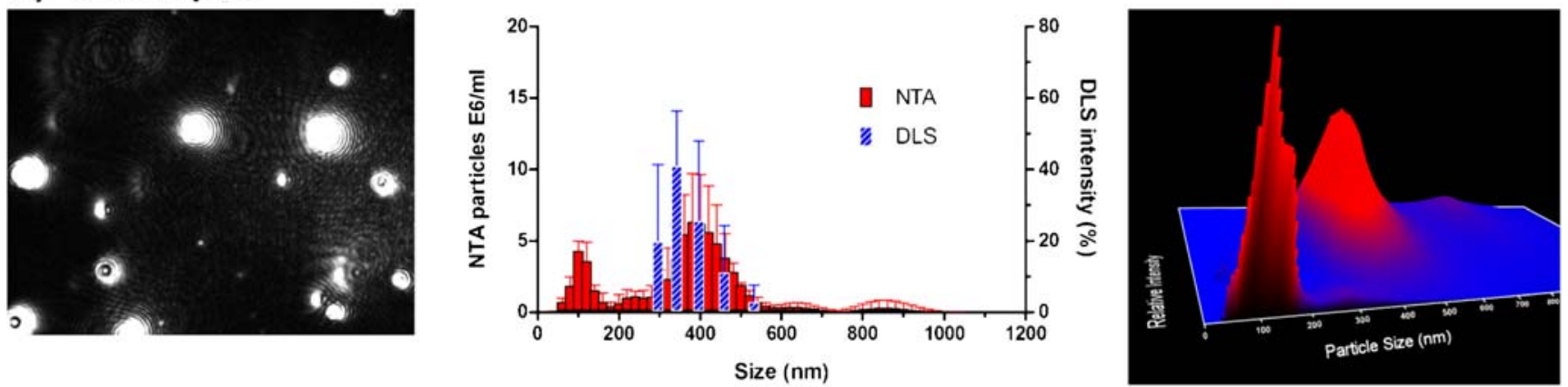

\section{c) Substantial spike}
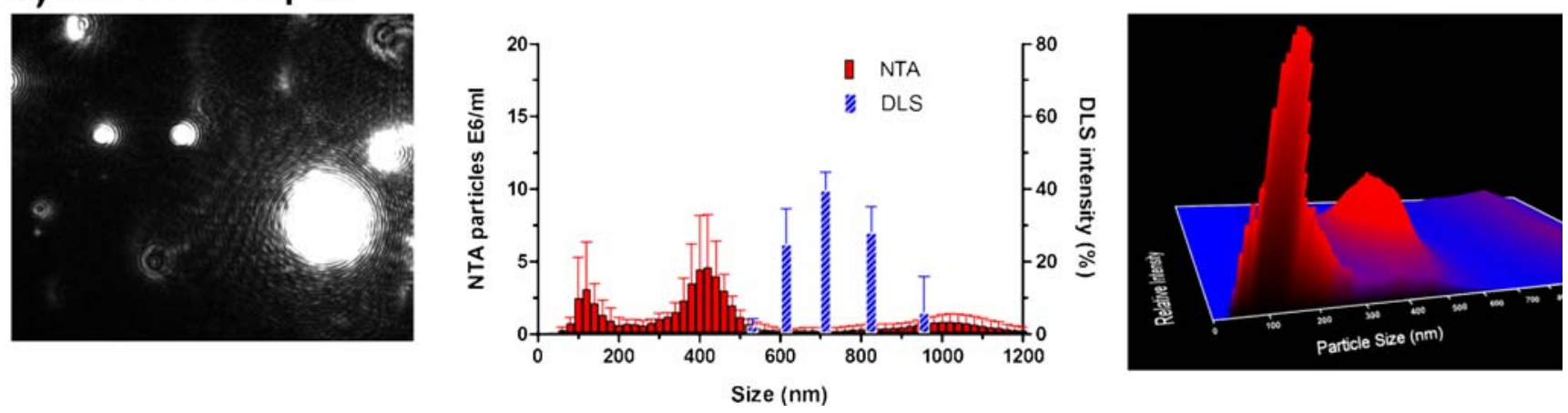

Fig. 4. Influence of large particles (1,000-nm beads) in a mixture of 100-nm and 400-nm monodisperse beads on NTA and DLS measurements. The size distribution (middle panels) with the corresponding NTA video frame (left panels) and normalized 3D graph (size vs. intensity vs. concentration; right panels) are shown. a) no 1,000-nm beads; b) 1:267 number ratio of 1,000-nm beads to the other beads in the mixture; c) 1:13 number ratio of 1,000 -nm beads to the other beads in the mixture. 


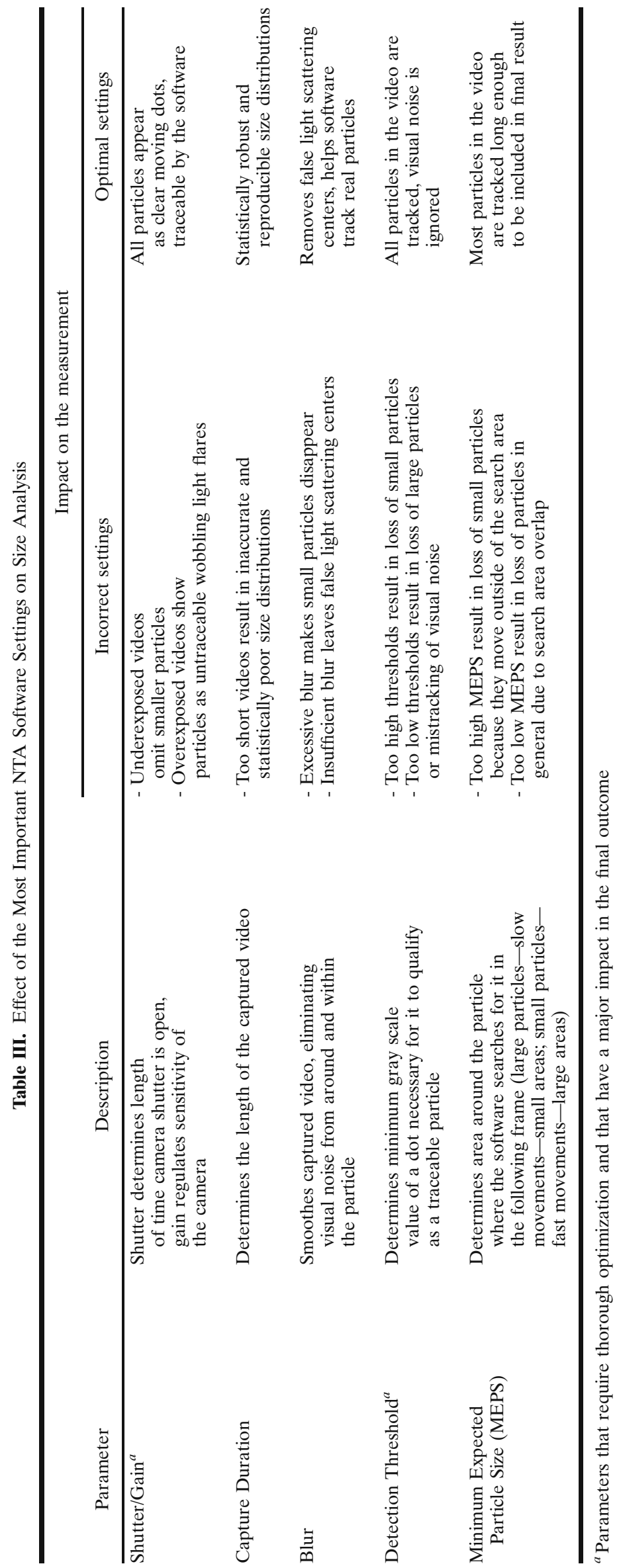


a) TMC

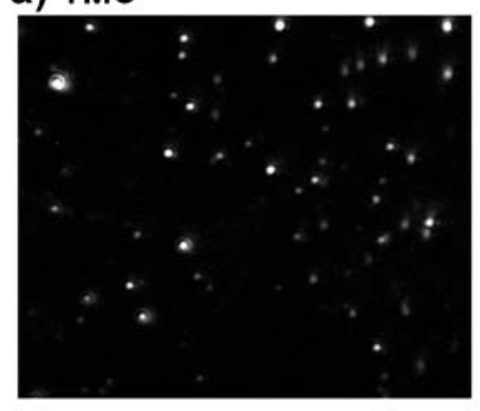

b) PLGA

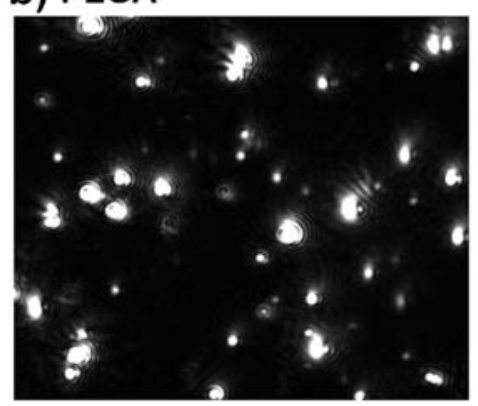

\section{c) Liposomes}

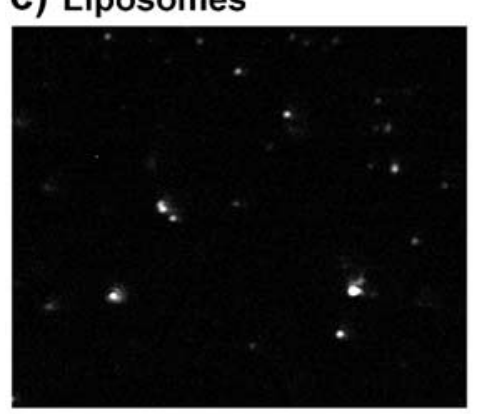

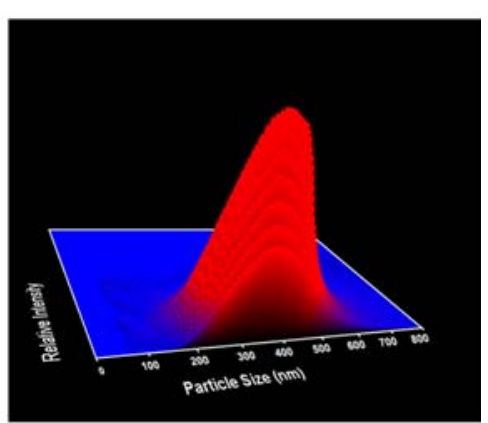

Size $(\mathrm{nm})$
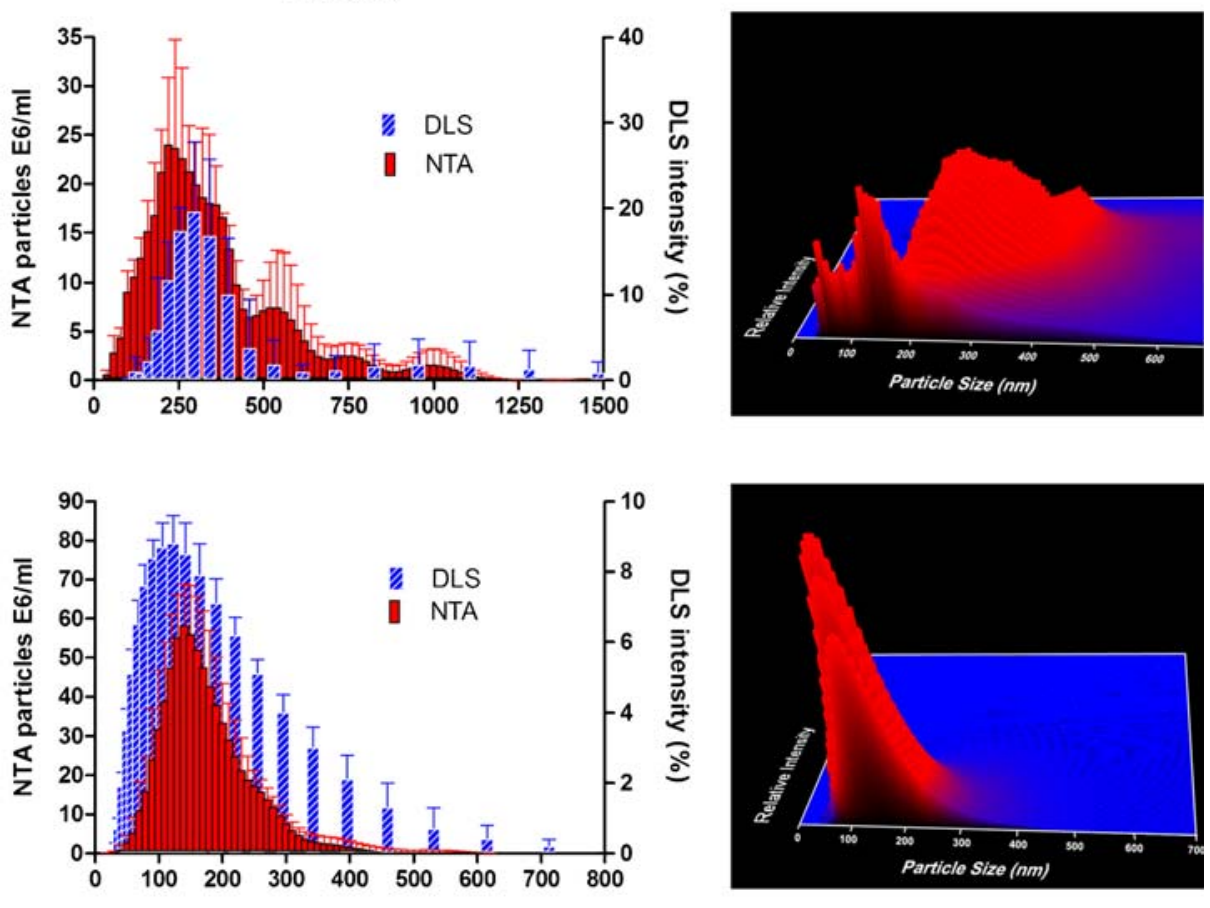

Fig. 5. Drug delivery nanoparticles measured with NTA and DLS. The size distribution (middle panels) with the corresponding NTA video frame (left panels) and 3D graph (size vs. intensity vs. concentration; right panels) are shown.

The lower PdI of this spiked sample is most likely a consequence of the masking effect of the $1,000 \mathrm{~nm}$ beads over the smaller beads in DLS measurements. These DLS values could have suggested that it was a fairly monodisperse $700 \mathrm{~nm}$ sample, when in fact it contained three distinct size populations of 100, 400 and $1,000 \mathrm{~nm}$. Such misinterpretation was not made when using NTA, since the presence of different populations was clearly detected by sample visualization. Although the big spike made the NTA measurements considerably more complex, all the beads in the sample could still be visualized and accurately sized (Fig. 4c). However, also in this case, spiking with large particles resulted in the underestimation of the number of smaller beads. After this spike, the number of $100-$ $\mathrm{nm}$ beads detected by NTA decreased by about $80 \%$ and that of the $400-n m$ beads by about $35 \%$.

\section{Effect of Settings in NTA Software on Particle Size Data}

As already mentioned, NTA involves several adjustment steps during the video capture and analysis, which are essential to obtain accurate measurement results. The power of choice given to the operator may be seen as a great advantage, but also raises concerns. The operator can easily choose settings that ignore or emphasize the presence of certain particles, which makes the veracity of the results dependent on individual judgment and experience.

To obtain accurate results, one should (i) thoroughly search with the microscope for the presence of all particle size classes in the sample, (ii) optimize the video settings in order to capture all these identified particle sizes, and (iii) adjust the analytical settings to unbiasedly track all moving particles captured by the video. With the purpose of clarifying the weight of the different software settings on the result, the influence of each parameter was carefully analyzed, as summarized in Table III.

Since the quality of NTA data will be dependent on the software settings used, which in turn depend on sample properties, as well as on the experience and decisions of the operator, NTA will be very difficult to qualify as a quality control method. Instead, NTA is_-like DLS—very useful as a characterization tool, as will be demonstrated in the applications discussed below. 


\section{a) $\lg G$}
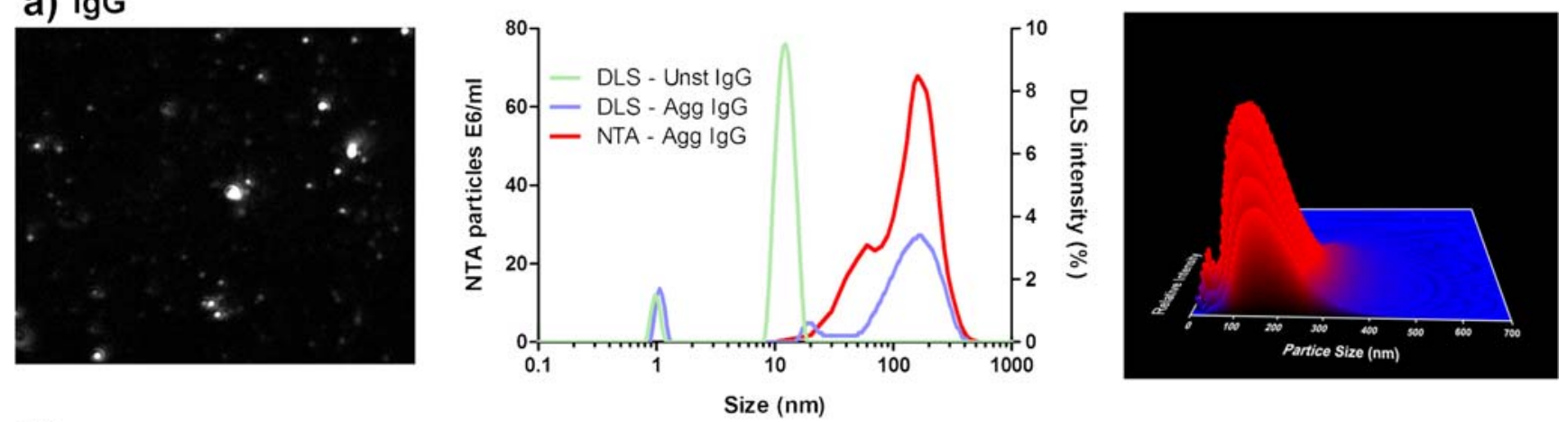

\section{b) Insulin}
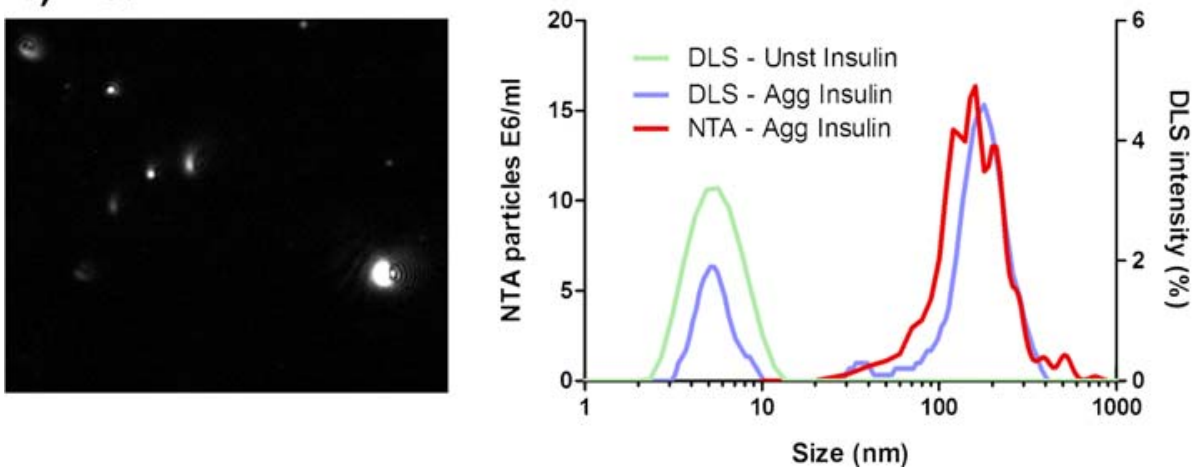

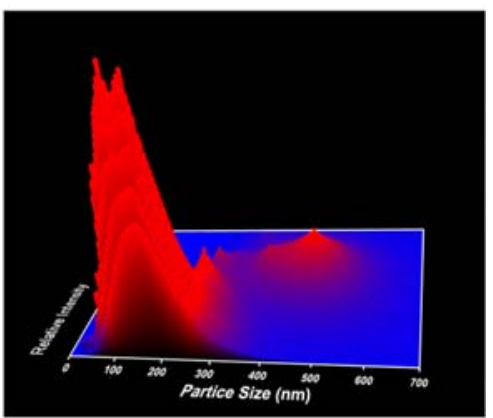

Fig. 6. IgG aggregates (obtained by heat stress) and insulin aggregates (obtained by metal catalyzed oxidation) measured with NTA and DLS. The size distribution (middle panels) with the corresponding NTA video frame (left panels) and 3D graph (size vs. intensity vs. concentration; right panels) are shown.

\section{NTA Applications}

\section{Drug Delivery Nanoparticles}

In order to evaluate the analytical performance of NTA for nanoparticles commonly used in the pharmaceutical field, PLGA particles, TMC particles and liposomes were analyzed with NTA and the results compared to DLS (Fig. 5).

DLS analysis resulted in a Z-ave of $411 \mathrm{~nm}$ and a PdI of 0.09 for the TMC particles, indicating a relatively monodisperse sample. This was confirmed by the visualization of the particles in the NanoSight sample chamber (Fig. 5a) and by the relatively low standard deviation $(91 \mathrm{~nm})$ given by NTA. However, the mean size obtained by NTA was $320 \mathrm{~nm}$, which is about $90 \mathrm{~nm}$ smaller than the Z-ave given by DLS, which points to a certain degree of polydispersity. The systematic size distribution shift towards larger sizes by DLS is more accentuated for the TMC particles than for the monodisperse polystyrene beads. This shift can be explained by the fact that size distributions obtained by DLS are intensity distributions, whereas NTA provides number distributions, which results in a larger shift in case of higher polydispersity.

The PLGA particles analyzed by DLS exhibited a Z-ave of $308 \mathrm{~nm}$ and a PdI of 0.22 . This PdI value suggests that the PLGA particles were more polydisperse than the TMC particles. The relatively high polydispersity of the PLGA particles became very clear during the visualization of the sample by NTA (Fig. 5b) and was confirmed by the high standard deviation (182 nm) obtained for the PLGA particles. The main population of these particles given by DLS was shifted to larger sizes as compared to NTA. However, contrary to most samples analyzed in this evaluation, the mean value observed with NTA (322 nm) was slightly higher than the Zave given by DLS (Fig. 5). This may be due to the inherent difficulty for DLS to properly analyze polydisperse samples.

DLS analysis of the liposomes resulted in a Z-ave of $117 \mathrm{~nm}$ and a PdI of 0.248 , suggesting that this sample was more polydisperse than the PLGA sample. Surprisingly, the visualization of the liposomes with NTA revealed a fairly monodisperse sample, and the standard deviation obtained was $77 \mathrm{~nm}$, which is even smaller than that of TMC particles. The mean size value obtained with NTA was $154 \mathrm{~nm}$, which is again larger than the Z-ave given by DLS. This time the peak given by DLS is shifted to smaller sizes as compared to the one obtained by NTA. Given that DLS has a lower detection limit than NTA, it is possible that smaller particles $(<30 \mathrm{~nm})$ present in the formulation decreased the Z-ave in DLS, which would also explain the relatively high PdI. Other analytical techniques would have been necessary to clarify this observation.

\section{Protein Aggregates}

A heat-stressed IgG formulation and a metal-oxidized insulin formulation were used to evaluate the analytical performance of NTA with protein aggregates (Fig. 6). The difference in the lower detection limits of the two techniques was evident when it came to the characterization of protein aggregates. DLS was able to detect not only the monomeric IgG $(\sim 11 \mathrm{~nm})$ but also the sucrose molecules $(\sim 1 \mathrm{~nm})$ present in the buffer, as was earlier described by others $(20,21)$. Given that the lower detection limit of NTA for proteins is about $30 \mathrm{~nm}$, protein monomers and aggregates smaller than this 

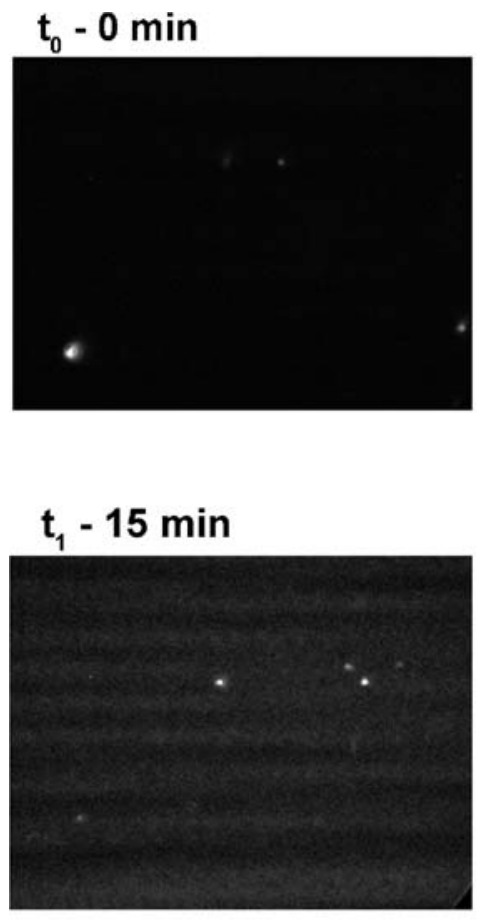

\section{$t_{2}-25 \min$}

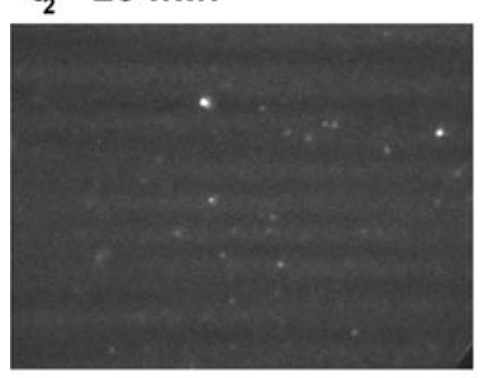

\section{$\mathbf{t}_{3}-35 \mathrm{~min}$}
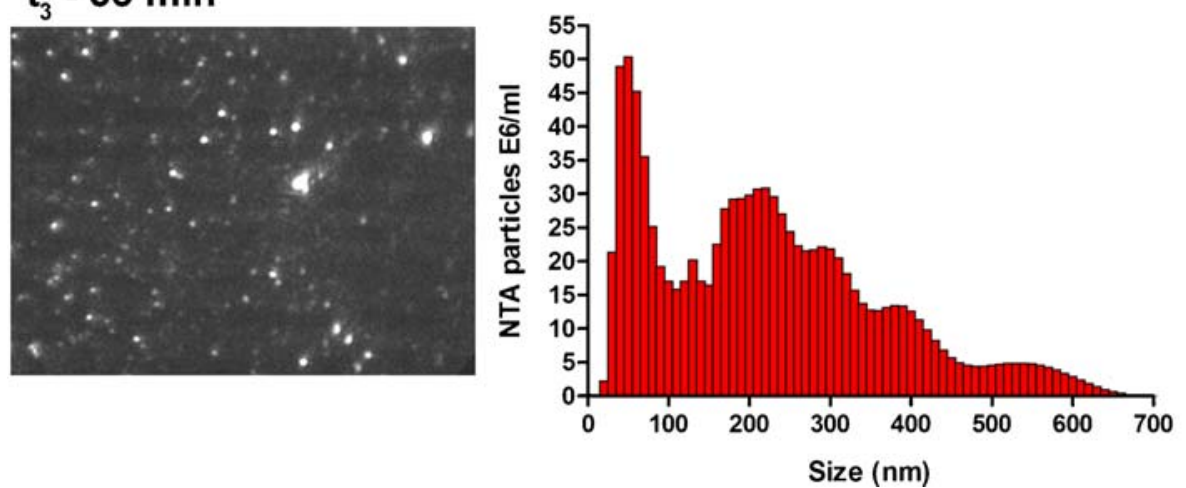
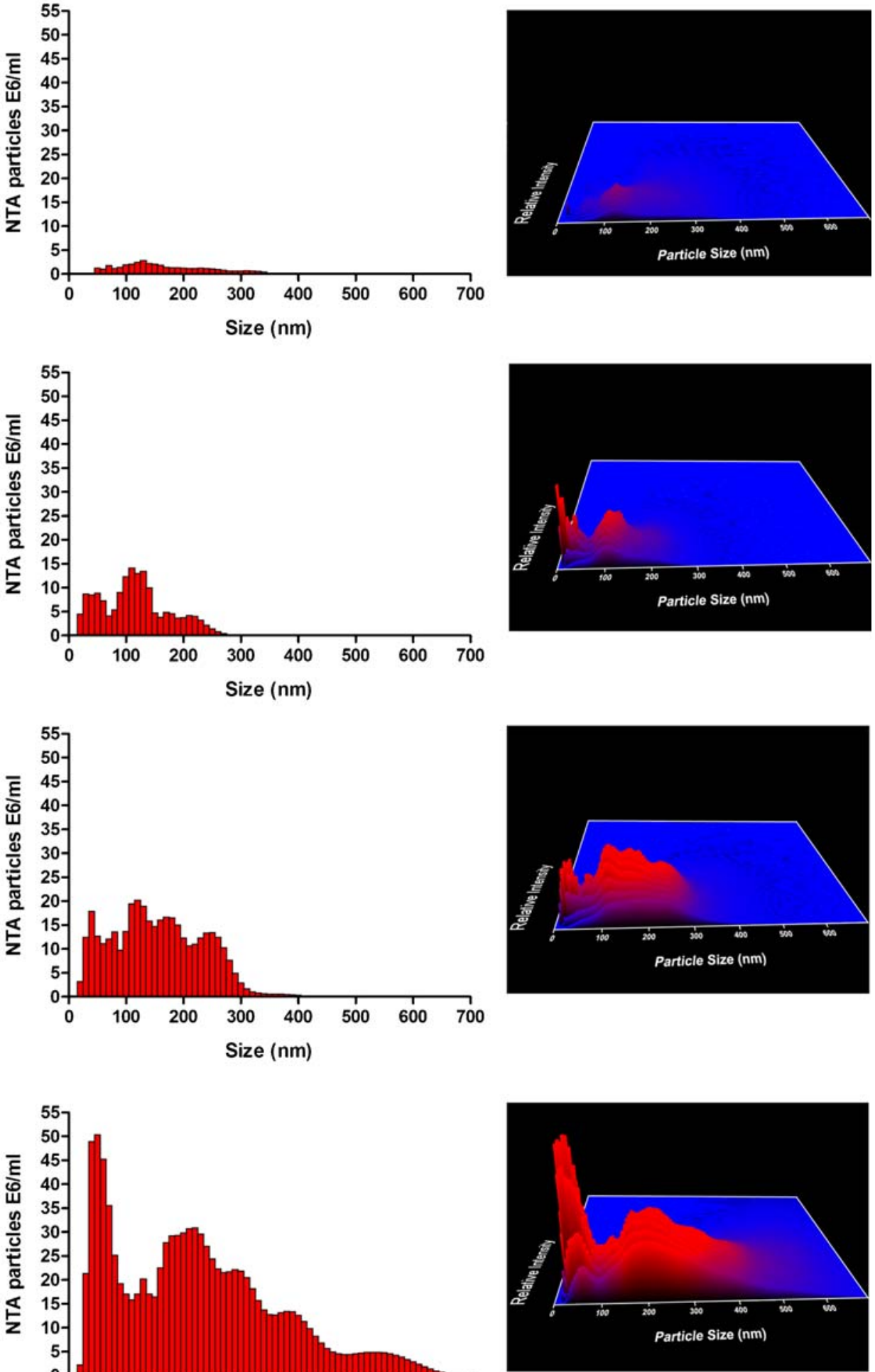

Fig. 7. Live monitoring of $\operatorname{IgG}$ aggregation at $50^{\circ} \mathrm{C}$ in the NanoSight sample chamber. The size distribution (middle panels) with the corresponding NTA video frame (left panels) and 3D graph (size $v s$. intensity $v s$. concentration; right panels) are shown.

are not detected by this technique. Nevertheless, in the stressed $\mathrm{IgG}$ formulation, the $\mathrm{IgG}$ aggregate size distribution obtained by DLS and NTA was similar, with a main peak at around $200 \mathrm{~nm}$. The Z-ave obtained by DLS was $47 \mathrm{~nm}$ and the PdI 0.5, while the average size according to NTA was
$175 \mathrm{~nm}$ and the standard deviation $76 \mathrm{~nm}$. The difference in the mean size values is most likely due to the fact that DLS considers aggregates, monomer and sucrose for the Z-ave (also explaining the high PdI), while NTA considers only aggregates for calculating the mean particle size (Fig. 6a). 
The visualization of the aggregated $\mathrm{IgG}$ sample with NTA allowed the distinction of two or more light scattering centers in very large aggregates $(>1 \mu \mathrm{m})$, which suggests that they were formed by the assembly of smaller aggregates. However, the brightness of such large particles interferes with the optimization of the instrument settings, because it makes smaller aggregates more difficult to detect. The monomer present in the sample increases the background light and also makes smaller aggregates more difficult do detect.

In the aggregated insulin sample, the native insulin was only detected by DLS and found to have an average size of about $6 \mathrm{~nm}$, consistent with literature data for insulin at neutral $\mathrm{pH}$ (22). Also for this sample, the aggregate size distribution was consistent between the two techniques, with a broad peak centered around $160 \mathrm{~nm}$ (Fig. 6b). The Z-ave given by DLS was $70 \mathrm{~nm}$ and the PdI 1.0, while the mean given by NTA was $199 \mathrm{~nm}$ and the standard deviation $103 \mathrm{~nm}$. Such a high PdI given by DLS suggested that the aggregates in the sample were very polydisperse, which can be confirmed by sample visualization and high standard deviation provided by NTA. Once again, the difference in mean value and $\mathrm{Z}$-ave given by the two techniques is most likely due to the fact that DLS considers native insulin and aggregates, while NTA considers only aggregates.

Table IV. Comparison of NTA with DLS

\begin{tabular}{|c|c|c|}
\hline & DLS & NTA \\
\hline \multicolumn{3}{|l|}{ Characteristics } \\
\hline Size accuracy & $\begin{array}{l}\text { Accurate for monodisperse samples, inaccurate } \\
\text { for polydisperse samples }\end{array}$ & $\begin{array}{l}\text { Accurate for both monodisperse and } \\
\text { polydisperse samples }\end{array}$ \\
\hline Peak resolution & Low ( $>3$ fold difference in diameter) & High $(<0.5$ fold difference in diameter) \\
\hline Size range & Ca. $1-1,000 \mathrm{~nm}$ & Ca. $30-1,000 \mathrm{~nm}$ \\
\hline Concentration range (particles $/ \mathrm{ml}$ ) & Broad (about $10^{8}-10^{12}$ ) & Limited $\left(10^{7}-10^{9}\right)$ \\
\hline Population ratio & $\begin{array}{l}\text { Narrow range } \\
\text { Large influence on size accuracy and distribution }\end{array}$ & $\begin{array}{l}\text { Very broad range } \\
\text { Very little influence on size accuracy and } \\
\text { little influence on size distribution }\end{array}$ \\
\hline Reproducibility & More reproducible & Less reproducible \\
\hline Contaminations & Large particles can seriously compromise the results & $\begin{array}{l}\text { Dust, microorganisms or aggregates } \\
\text { easily detected, large particles have } \\
\text { little influence }\end{array}$ \\
\hline \multicolumn{3}{|l|}{ Operational } \\
\hline Device handling & $\begin{array}{l}\text { Very user-friendly } \\
\text { Little sample handling } \\
\text { Possibility of using disposable cuvettes }\end{array}$ & $\begin{array}{l}\text { Requires several parameter adjustments } \\
\text { Sample handling may affect size distribution } \\
\text { Sample chamber must be cleaned after each } \\
\text { sample } \\
\text { Experienced operator required }\end{array}$ \\
\hline Time consumption & Between 2 to 5 min per measurement & Between 5 min to 1 hour per measurement \\
\hline Particular output & $\begin{array}{l}\text { Approximate size distribution } \\
\text { Intensity distribution } \\
\text { Z-ave } \\
\text { PdI }\end{array}$ & $\begin{array}{l}\text { Individual particle sizing } \\
\text { Number distribution } \\
\text { Approximate concentrations } \\
\text { Individual particle intensity }\end{array}$ \\
\hline Sample visualization & No & Yes \\
\hline \multicolumn{3}{|l|}{ Applications } \\
\hline Drug delivery nanoparticles & $\begin{array}{l}\text { Accurate sizing } \\
\text { Approximate size distribution } \\
\text { Hard detection of contaminants }\end{array}$ & $\begin{array}{l}\text { Accurate sizing } \\
\text { More reliable size distribution } \\
\text { Easy detection of contaminants }\end{array}$ \\
\hline Protein aggregates & $\begin{array}{l}\text { Approximate size distribution } \\
\text { Includes protein monomers and some } \\
\text { excipients } \\
\text { Presence of very large aggregates has a } \\
\text { big impact on the result }\end{array}$ & $\begin{array}{l}\text { Accurate size distribution } \\
\text { Protein monomer and small aggregates } \\
\text { excluded } \\
\text { Aggregate architecture information } \\
\text { Interference of large aggregates may } \\
\text { sometimes be overcome }\end{array}$ \\
\hline
\end{tabular}


Several factors are known to induce protein aggregation, and some characterization techniques have been reported to induce or disturb the aggregation state (8). During NTA measurements, the sample is in contact with glass, stainless steel, a Viton fluoroelastomer O-ring and nylon tubing. Moreover, the samples are submitted to a slight shear during the injection into the measurement cell. It has been reported that the synergistic effect of adsorption of a monoclonal antibody to stainless steel and shear can create small amounts of aggregates (23). In fact, we noticed that some stressed $\mathrm{IgG}$ formulations slightly increase the amount and size of aggregates if left in the sample chamber for more than $30 \mathrm{~min}$ (data not shown). This phenomenon became more evident if the sample was moved back and forth in the measurement cell with the syringe piston. While DLS size measurements are usually performed in disposable (polystyrene) cuvettes, the NanoSight sample chamber has to be manually cleaned and reused. Furthermore, as previously mentioned, NTA measurements often require sample dilution, which may destroy or create new aggregates and affect the size distribution (8). In general, the effect of sample content dilution on the sample content is related to the instability of the submicron particles and is more likely to affect protein aggregates than drug delivery nanoparticles.

Overall, DLS sample treatment seems to be less aggressive than NTA, which is an advantage for unstable samples, such as protein aggregates. However, the high peak resolution and suitability for polydisperse samples make NTA a very useful technique to analyze protein aggregates.

\section{Live Monitoring of Heat-Induced Protein Aggregation}

The heating block of NanoSight allows NTA measurements at temperatures ranging from room temperature to $50^{\circ} \mathrm{C}$, which enables the live monitoring of protein aggregation at elevated temperatures. This feature is also possible with DLS, but the possibility of visualizing the aggregates being formed with NTA gives a more complete overview of the aggregation process.

An IgG formulation was heated at $50^{\circ} \mathrm{C}$ for $45 \mathrm{~min}$ in the NanoSight heating block, while movies were being recorded (Fig. 7). Since NTA is not capable of detecting particles smaller than about $30 \mathrm{~nm}$, it was not possible to see the formation of dimers, trimers or any small oligomers. At $t_{0}$, the sample had already been exposed to some heat stress for about $10 \mathrm{~min}$, the time required for the temperature to rise from room temperature to $50^{\circ} \mathrm{C}$, and some polydisperse aggregates of about $50-350 \mathrm{~nm}$ were detected. At $\mathrm{t}_{1}$, the number of aggregates had increased, and distinct subpopulations became apparent around $50 \mathrm{~nm}, 100 \mathrm{~nm}$ and $200 \mathrm{~nm}$. After about $20 \mathrm{~min}$, the number of aggregates rapidly increased. Even though the number of aggregates had slightly increased at $t_{2}$, it does not reflect this sudden increase of aggregates observed, because the background scattering (visible in the video frame of $\mathrm{t}_{2}$ ) made particle tracking more difficult for the NTA software. After $35 \mathrm{~min}$ of heat-stressing, the number of aggregates reached the upper concentration limit of NTA, and especially the number of aggregates with sizes around $50 \mathrm{~nm}$ increased significantly. After $45 \mathrm{~min}$, very large $(>5 \mu \mathrm{m})$ aggregates were visible, and they made accurate NTA size measurements impossible, because the light they scattered masked the smaller traceable aggregates $(<1 \mu \mathrm{m})$. These large aggregates contained several intense light scattering centers (results not shown), which were probably formed by the assembly of several smaller aggregates. Unfortunately, aggregate assembly process was not detectable by this technique, since the aggregates were constantly entering and leaving the view area.

\section{CONCLUSIONS}

In this work, we evaluated NTA as a new characterization method for nanoparticle analysis and compared it to DLS. The differences between the two techniques are summed in Table IV. NTA can be time-consuming and requires some operational skills for the adjustment all software settings, but has some clear advantages over DLS. NTA enables the visualization of the sample, gives an approximate particle concentration and obtains size information based on the Brownian motion of individual particles. NTA is very accurate for sizing both monodisperse and polydisperse samples and has a substantially better peak resolution. The presence of few large particles in a sample has a little impact on NTA sizing accuracy, but reduces the number of small particles detected by the software. Different population ratios in standard polystyrene bead mixtures are easily detected and do not affect the sizing accuracy.

NTA proved to be very suitable for analyzing drug delivery nanoparticles. This technique is also very suitable for analyzing protein aggregates, but care should be taken that sample preparation does not influence the aggregate distribution in the measurement cell. It also gives the possibility of live monitoring heat-induced aggregation, providing information about the aggregation kinetics.

\section{AKNOWLEDGEMENTS}

This research is supported by the Dutch Technology Foundation STW, applied science division of NWO and the Technology Program of the Ministry of Economic Affairs. The authors are grateful to Bram Slütter and Ana Silva for the preparation of the TMC nanoparticles and PLGA particles, to Myrra Carstens for the preparation of the liposomes and to Riccardo Torosantucci and Olubukayo Oyetayo for preparing the aggregated protein samples.

Open Access This article is distributed under the terms of the Creative Commons Attribution Noncommercial License, which permits any noncommercial use, distribution, and reproduction in any medium, provided the original author(s) and source are credited.

\section{REFERENCES}

1. Mahato RI, Narang AS, Thoma L, Miller DD. Emerging trends in oral delivery of peptide and protein drugs. Crit Rev Ther Drug Carrier Syst. 2003;20:153-214.

2. Lipinski CA. Drug-like properties and the causes of poor solubility and poor permeability. J Pharmacol Toxicol Meth. 2000;44:235-49. 
3. Jiskoot W, van Schie RM, Carstens MG, Schellekens H. Immunological risk of injectable drug delivery systems. Pharm Res. 2009;26:1303-14.

4. Cromwell ME, Hilario E, Jacobson F. Protein aggregation and bioprocessing. AAPS J. 2006;8:E572-9.

5. Schellekens H. Bioequivalence and the immunogenicity of biopharmaceuticals. Nat Rev Drug Discov. 2002;1:457-62.

6. Mahler HC, Friess W, Grauschopf U, Kiese S. Protein aggregation: pathways, induction factors and analysis. J Pharm Sci. 2009;98:2909-34.

7. Frokjaer S, Otzen DE. Protein drug stability: a formulation challenge. Nat Rev Drug Discov. 2005;4:298-306.

8. Philo JS. A critical review of methods for size characterization of non-particulate protein aggregates. Curr Pharm Biotechnol. 2009;10:359-72.

9. Carpenter JF, Randolph TW, Jiskoot W, Crommelin DJ, Middaugh $\mathrm{CR}$, Winter G, et al. Overlooking subvisible particles in therapeutic protein products: gaps that may compromise product quality. J Pharm Sci. 2009;98:1201-5.

10. Bootz A, Vogel V, Schubert D, Kreuter J. Comparison of scanning electron microscopy, dynamic light scattering and analytical ultracentrifugation for the sizing of poly(butyl cyanoacrylate) nanoparticles. Eur J Pharm Biopharm. 2004;57:369-75.

11. Brown PH, Schuck P. Macromolecular size-and-shape distributions by sedimentation velocity analytical ultracentrifugation. Biophys J. 2006;90:4651-61.

12. Fraunhofer W, Winter G, Coester C. Asymmetrical flow field-flow fractionation and multiangle light scattering for analysis of gelatin nanoparticle drug carrier systems. Anal Chem. 2004;76:1909-20.

13. Frisken BJ. Revisiting the method of cumulants for the analysis of dynamic light-scattering data. Appl Opt. 2001;40:4087-91.

14. Demeester J, Smedt S, Sanders N, Haustraete J. Light Scattering. In: Jiskoot W, Crommelin DJ, editors. Methods for structural analysis of protein pharmaceuticals. Arlington: AAPS; 2005.

15. Berne B, Pecora R. Dynamic light scattering with applications to chemistry, biology, and physics. Mineola: Dover; 2000.

16. Applications of Nanoparticle Tracking Analysis (NTA) in Nanoparticle Research. http://www.schaefer-tec.com/fileadmin/user upload/sortiment/nanopartikel/NanoSight/NANOSIGHT_Appli cation_Review_NTA_April_2009_M201B.pdf (accessed 26/09/ 2009), part of NanoSight. http://www.nanosight.co.uk (accessed 26/09/2009).

17. Slutter B, Plapied L, Fievez V, Sande MA, des Rieux A, Schneider YJ, et al. Mechanistic study of the adjuvant effect of biodegradable nanoparticles in mucosal vaccination. J Control Release. 2009;138:113-21.

18. Minimum DLS concentration? http://www.malvern.com/malvern/ kbase.nsf/allfaqbyno/KB000795?opendocument (accessed 22/09/ 2009), part of Malvern support FAQ. http://www.malvern.com (accessed 22/09/2009).

19. Can DLS resolve oligomer mixtures? http://www.malvern.com/ malvern/kbase.nsf/allfaqbyno/KB001102 (accessed 22/09/2009), part of Malvern support FAQ. http://www.malvern.com (accessed 22/09/2009).

20. Mahler HC, Muller R, Friess W, Delille A, Matheus S. Induction and analysis of aggregates in a liquid IgG1-antibody formulation. Eur J Pharm Biopharm. 2005;59:407-17.

21. Kaszuba M, McKnight D, Connah MT, McNeil-Watson FK, Nobbmann U. Measuring sub nanometre sizes using dynamic light scattering. J Nanopart Res. 2007;10:823-9.

22. Hvidt S. Insulin association in neutral solutions studied by light scattering. Biophys Chemist. 1991;39:205-13.

23. Bee JS, Stevenson JL, Mehta B, Svitel J, Pollastrini J, Platz R, et al. Response of a concentrated monoclonal antibody formulation to high shear. Biotechnol Bioeng. 2009;103:936-43. 